\title{
Does Implant Density Impact Three-Dimensional Deformity Correction in Adolescent Idiopathic Scoliosis with Lenke 1 and 2 Curves Treated by Posterior Spinal Fusion without Ponte Osteotomies?
}

\author{
Thamrong Lertudomphonwanit ${ }^{1}$, Chirag A. Berry ${ }^{2}$, Viral V. Jain ${ }^{3}$, Peter F Sturm ${ }^{3}$ \\ ${ }^{1}$ Department of Orthopaedics, Faculty of Medicine, Ramathibodi Hospital, Mahidol University, Bangkok, Thailand \\ ${ }^{2}$ Cincinnati VA Medical Center, Cincinnati, OH, USA \\ ${ }^{3}$ Division of Orthopaedic Surgery, Cincinnati Children's Hospital Medical Center, Cincinnati, OH, USA
}

Study Design: Retrospective cohort study.

Purpose: To determine whether implant density impact three-dimensional deformity correction in posterior spinal fusion (PSF) without Ponte osteotomies (POs) for patients with Lenke 1 and 2 adolescent idiopathic scoliosis (AIS).

Overview of Literature: Currently, the optimal pedicle screw (PS) density for flexible moderate-sized thoracic AIS curve correction is still controversial. There are limited data regarding the impact of implant density on three-dimensional correction in PSF without the use of $\mathrm{PO}$ for thoracic AIS surgery.

Methods: A database of patients with AIS with Lenke 1 and 2 curves treated with PSF without P0 and instrumented with PSs and $\geq 2$-year follow-up was reviewed. The preoperative, immediate, and final follow-up postoperative radiographs were analyzed. The correlation between PS density and the following factors were determined: major curve correction (MCC), correction index (Cl; MCC/ curve flexibility), kyphosis angle change, and rib index (RI) correction. Then, patients were divided into low-density (LD) and highdensity (HD) groups according to mean PS density for the entire cohort (1.5 PS per level). Demographics and radiographic and clinical outcomes were compared between groups.

Results: The study included 99 patients with Lenke 1 and 23 patients with Lenke 2 AIS. The average MCC was $67.2 \%$. There was no correlation between screw density and these parameters: MCC $(r=0.10, p=0.26), \mathrm{Cl}(r=0.16, p=0.07)$, change in T2-T12 kyphosis angle $(r=-0.13, p=0.14)$, and RI correction $(r=-0.09, p=0.37)$. Demographic and preoperative radiographic parameters were similar between the $L D$ and $\mathrm{HD}$ groups. At the latest follow-up, there were no differences between the two groups in regard to $\mathrm{MCC}$, $\mathrm{Cl}$, change in T2-T12 kyphosis angle, RI correction, and Scoliosis Research Society-30 scores (all $p>0.05$ ).

Conclusions: This study revealed no significant correlation between screw density and curve correction in any planes. HD construct may not provide better deformity correction in patients with flexible and moderate thoracic AIS undergoing PSF without PO.

Keywords: Adolescent idiopathic scoliosis; Pedicle screws; Implant density; Curve correction

Received Jul 25, 2020; Revised Dec 4, 2020; Accepted Dec 14, 2020

Corresponding author: Viral V. Jain

Division of Orthopaedic Surgery, Cincinnati Children's Hospital Medical Center, 3333 Burnet Avenue, MLC 2017, Cincinnati, OH, 45229, USA

Tel: +1-513-636-7060, Fax: +1-513-636-3928, E-mail: Viral.Jain@cchmc.org

*The investigation was performed at Division of Orthopaedic Surgery, Cincinnati Children's Hospital Medical Center. 


\section{Introduction}

Pedicle screw (PS) constructs have become the standard instrumentation for posterior correction and spinal fusion of adolescent idiopathic scoliosis (AIS) [1]. PS instrumentation can improve three-dimensional spinal deformity correction and solid fusion to achieve the goal of surgical treatment of AIS [2]. In patients with thoracic AIS, PS constructs have been shown to improve radiographic outcomes compared with hook or hybrid constructs [3-5]. By providing rigid three-column fixation of the vertebra, PS constructs have been shown to obviate the need for anterior fusion, decrease the number of fusion levels, reduce complication and revision rates, and improve pulmonary function values $[3,6,7]$.

PS density is defined as the number of PS per fused level. Many surgeons use high PS density (HD) constructs with maximum density of 2.0 PS per fused level despite limited data to show the optimal PS density constructs [810]. There are valid concerns of HD constructs, including a small but significant risk of PS malposition, increased cost, and postoperative hypokyphosis $[8,11,12]$. If low PS density (LD) constructs are able to improve curve correction comparable to HD constructs, using fewer screws would improve the efficiency and reduce the implant costs of scoliosis surgery. Although PS constructs have been commonly used in the treatment of thoracic AIS, the PS density for optimal outcomes is still controversial. Some studies have demonstrated larger radiographic correction with HD $[8,9]$. On the contrary, several studies have shown no correlation between PS density and radiographic correction [12-14]. There are many conflicting factors that can affect the surgical results on curve correction, including curve magnitude, curve flexibility, number and points of fixation, fixation apparatus, and posterior release procedure (i.e., Ponte osteotomy) $[15,16]$. To the best of our knowledge, no previous studies reported the impact of PS density on deformity correction in posterior spinal fusion (PSF) without the use of additional posterior release procedure especially for Ponte osteotomies (POs). Furthermore, the optimal PS density remains unknown, especially when sagittal and axial correction is considered.

This study aimed to determine whether the number of screws per fused level, or PS density, affects coronal curve correction, thoracic kyphosis, and apical vertebral rotation in PSF without POs of Lenke 1 and 2 AIS and investigate whether HD improves three-dimensional deformity correction at the final follow-up.

\section{Materials and Methods}

\section{Patient identification}

This retrospective study was approved by the institutional review board of Cincinnati Children's Hospital Medical Center (CCHMC 2014-7694). A database of consecutive patients with AIS who underwent posterior instrumented spinal fusion from a single institution between 2006 and 2013 was reviewed. The requirement for informed consent from individual patients was omitted because of the retrospective design of this study. The inclusion criteria were diagnosis of Lenke curve type 1 and 2 AIS, age between 10 and 20 years, preoperative thoracic curve between $45^{\circ}$ and $80^{\circ}$ and preoperative curve flexibility $>25 \%$, surgical procedure of single-stage PSF in which at least $75 \%$ of the anchors were screws, and minimum of 2 years' follow-up. Patients who had a history of spinal surgery, had abnormal spinal cord signal, or underwent additional posterior release procedures, such as POs, were excluded. Since POs performed in thoracic AIS curves may affect flexibility and curve correction [16], we included patients who had only preoperative thoracic curve between $45^{\circ}$ and $80^{\circ}$ and preoperative curve flexibility $>25 \%$ and did not undergo POs at the index procedure to eliminate this confounding factor on curve correction.

\section{Surgical technique}

Patients were placed in the prone position on the operating table. A posterior midline incision and subperiosteal dissection were made to the tips of transverse process at all levels of spinal fusion. PSs were inserted with a free-hand technique. Fusion levels were selected as described by Lenke et al. [17]. The lower instrumented vertebra (LIV) was a neutral lumbar vertebra touched by the central sacral vertical line on coronal radiographs for modifier A curves and proximal stable vertebra (distal end of the thoracic curve) for modifier $\mathrm{B}$ and $\mathrm{C}$ curves. The upper instrumented vertebra (UIV) was determined by the rigidity of the proximal thoracic curve and T1 tilt and should be above the apex of the thoracic kyphosis. Regarding the placement of screws in patients with LD, PSs were placed bilaterally at two UIV, two LIV, and the apical vertebra and its two adjacent vertebrae, if available (Fig. 1). The philosophy for selection of 

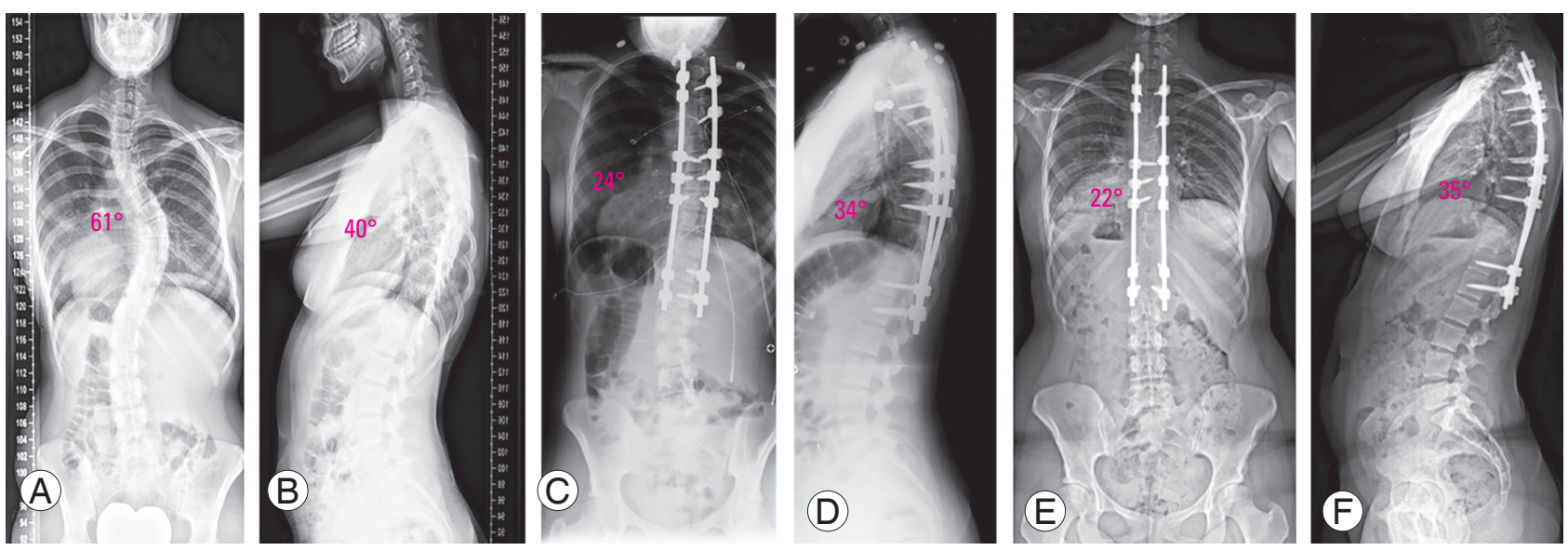

Fig. 1. Illustrative example of a low-screw density construct, pedicle screw density is 1.17. (A, B) Preoperative radiographs of a 15-year-0ld female patient with adolescent idiopathic scoliotic curve (T5-T11: $61^{\circ}$, thoracic kyphosis: $40^{\circ}$ ). (C, D) Immediate postoperative standing radiographs demonstrated a correction in Cobb angle to $24^{\circ}$, and thoracic kyphosis measured $34^{\circ}$. (E, F) Last follow-up radiographs, 3.5 years after surgery, the coronal Cobb angle and thoracic kyphosis were well maintained during the follow-up period.
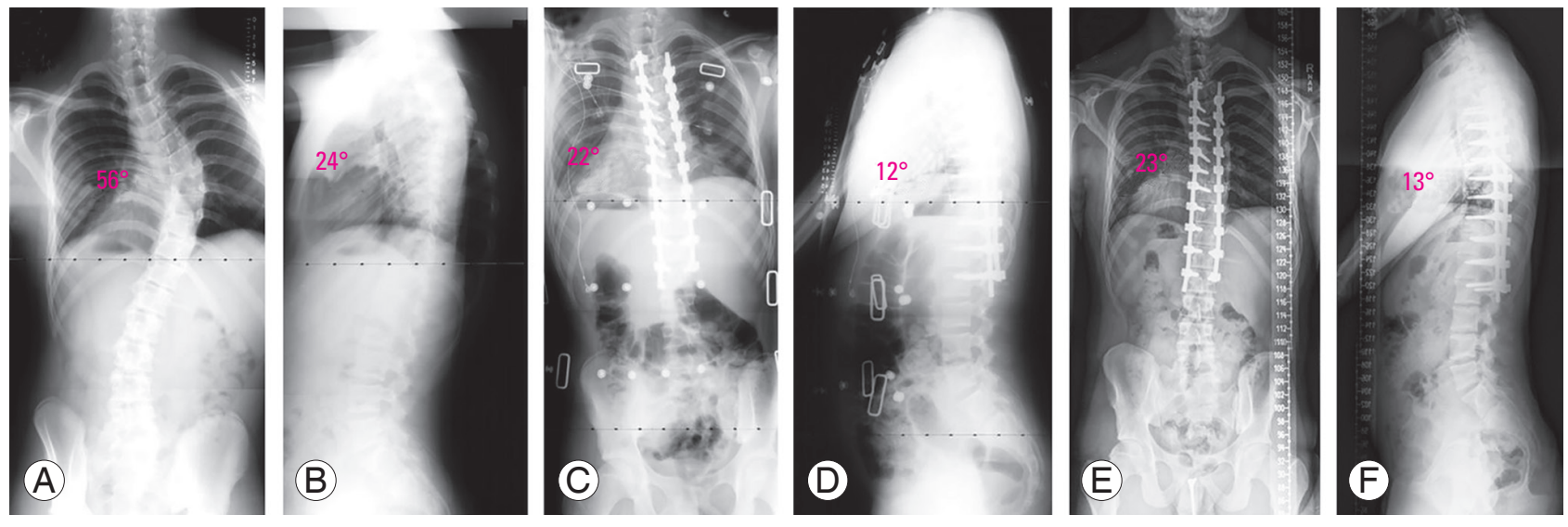

Fig. 2. Illustrative example of a high-screw density construct, pedicle screw density is 1.82. (A, B) Preoperative radiographs of a 15-year-old male patient with adolescent idiopathic scoliotic curve (T4-T11: $56^{\circ}$, thoracic kyphosis: $2^{\circ}$ ). (C, D) Immediate postoperative standing radiographs demonstrated a correction in Cobb angle to $22^{\circ}$, and thoracic kyphosis measured $12^{\circ}$. (E, F) Last follow-up radiographs, 4 years after surgery, the coronal Cobb angle and thoracic kyphosis were well maintained during the follow-up period.

implant levels is stable base, stable proximal construct, and apical control. Apical control was achieved by concave side screws and alternate convex side screw or with bilateral apical only screws. In patients with HD, PSs were placed bilaterally at all fused levels, if available (Fig. 2) [17]. One surgeon was involved in each index procedure. Regarding the deformity correction maneuvers, same rod rotation and direct vertebral rotation techniques were performed in all patients. Additional correction was performed by slight concave distraction and convex compression, if necessary. No additional flexibility-modifying procedure (i.e., Ponte osteotomy) was performed.

\section{Radiographic and clinical outcome measurements}

An independent spine surgeon performed all radiographic and clinical measurements. Radiographic analysis included preoperative, 1-week postoperative, and final follow-up major Cobb angle, T2-T12 kyphosis angle, and preoperative supine side bending major Cobb angle. Rib hump deformity and vertebral rotation were evaluated using rib index (RI) method and extracted from double rib contour sign on standing lateral radiographs in patients who did not undergo costoplasty (97 patients) [18]. The RI was calculated by the ratio of spine distances $\mathrm{d} 1 / \mathrm{d} 2$. $\mathrm{d} 1$ is the distance between the most extended point of the 


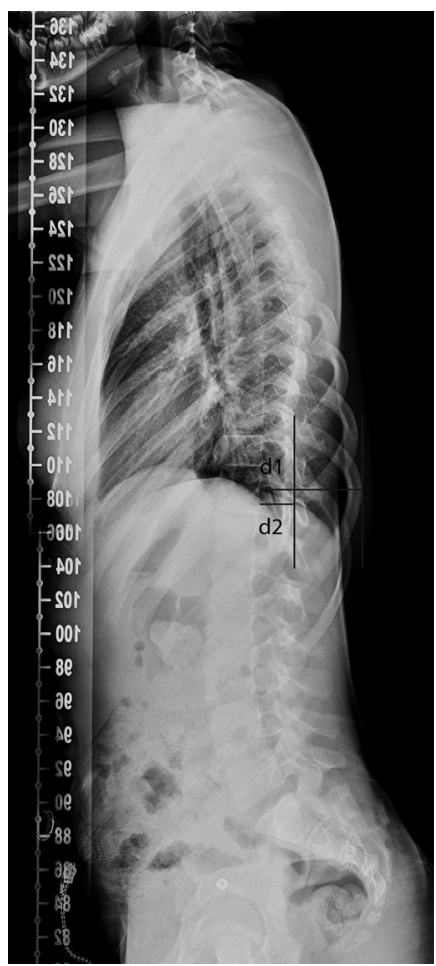

Fig. 3. Lateral standing radiograph illustrating the rib index (RI) using the double rib contour sign. $\mathrm{Rl}$ equals $\mathrm{d} 1 / \mathrm{d} 2$. $\mathrm{d} 1$ is the distance between the most extended point of the most prominent rib contour and the posterior margin of the corresponding vertebra, $\mathrm{d} 2$ is the distance from the posterior margin of the same vertebra and the least prominent rib contour.

most projecting rib contour and the posterior margin of the corresponding vertebra. $\mathrm{d} 2$ is the distance from the posterior margin of the same vertebra and the least projecting rib contour (Fig. 3). Preoperative curve flexibility was defined as (preoperative Cobb angle-side bending Cobb angle) $/($ preoperative Cobb angle $) \times 100 \%$. Percent major curve correction (MCC) was defined as (preoperative major Cobb angle-postoperative major Cobb angle)/ (preoperative major Cobb angle) $\times 100 \%$. Correction index (CI) was defined as (major Cobb correction)/(preoperative curve flexibility). CI was used to eliminate the effect of flexibility on the correction as described by Vora et al. [19]. Percent RI correction was defined as (preoperative RIpostoperative RI)/(preoperative RI) $\times 100 \%$. The PS density of each construct was calculated as total numbers of PSs/ number of fused levels.

Medical records were reviewed to obtain demographic data and the following perioperative data: number of fused levels, number of total PSs in construct, number of PSs per instrumented level, number of other anchors in construct, implant information (material and diameter of rod use), estimated blood loss, operative time, and complications. Patient-reported outcomes were assessed using Scoliosis Research Society (SRS)-30 questionnaire at the latest follow-up.

\section{Statistical analysis}

Data were analyzed using Stata ver. 14.0 software (StataCorp LP, 2015; Stata Corp., College Station, TX, USA). We performed power analysis and found that our sample size $(n=122)$ achieved a statistical power of 0.71 with a significance of 0.05 and correlation coefficient of 0.21 . Pearson correlation analysis was used to determine the correlation between PS density and the following parameters: percent MCC and CI, change in T2-T12 kyphosis, and percent RI correction. The correlation between curve flexibility and percent MCC was also determined. Subgroup correlation analyses were also performed in regard to Lenke curve type (Lenke- 1 curve type and Lenke- 2 curve type), patient age, (patients with age $>15$ years and patients with age $<15$ years), Risser sign (Risser grade $<3$ and Risser grade $\geq 3$ ), and body mass index (BMI $<25 \mathrm{~kg} / \mathrm{m}^{2}$ and BMI $\geq 25$ $\mathrm{kg} / \mathrm{m}^{2}$ ). Then, patients were divided into two groups according to the mean PS density for the entire study (1.50 screws per level). Thus, LD construct was defined as the use of $<1.50$ screws per level fused, and HD construct was defined as the use of 1.50 or more screws per fused level. Independent sample $t$-tests were used to compare demographic data, radiographic parameters, and clinical outcomes at baseline and follow-up between the two groups. Quantile regression tests were used to compare change in T2-T12 kyphosis angle and loss-correction rate between the two groups. Significance was set at a $p$-value $<0.05$.

\section{Results}

The study included 122 patients: 99 patients with Lenke 1curve type and 23 patients with Lenke 2 curve type. There were 106 female and 16 male patients with a minimum follow-up of 2 years (average, 40.1 months; range, 24-92 months). The demographic characteristics and clinical features of the patients are shown in Table 1. The mean \pm standard deviation age at surgery was $15.0 \pm 1.9$ years. The median Risser grade of the patients was 3 (range, $0-5$ ). The majority (54.9\%) of patients had Risser grade $\geq 3$. The mean preoperative major Cobb angle was $55.3^{\circ} \pm 7.2^{\circ}$ and corrected to $18.3^{\circ} \pm 7.2^{\circ}$ ) at follow-up 
Table 1. Demographic characteristics and clinical features of the patients

\begin{tabular}{|c|c|}
\hline Characteristic & Value \\
\hline Age at surgery (yr) & $15.0 \pm 1.9(11$ to 20$)$ \\
\hline Weight (kg) & $56.0 \pm 13.3$ (28.2 to 107$)$ \\
\hline Height (cm) & $161 \pm 9.3$ (133 to 193) \\
\hline Body mass index $\left(\mathrm{kg} / \mathrm{m}^{2}\right)$ & $21.8 \pm 5.1$ (15.1 to 48.8$)$ \\
\hline \multicolumn{2}{|l|}{ Risser sign } \\
\hline Grade 0 & $14(11.5)$ \\
\hline Grade 1 & $19(15.6)$ \\
\hline Grade 2 & $22(18.0)$ \\
\hline Grade 3 & $22(18.0)$ \\
\hline Grade 4 & $24(19.7)$ \\
\hline Grade 5 & $21(17.2)$ \\
\hline Median Risser grade & $3(0$ to 5$)$ \\
\hline No. of fused levels & $11.1 \pm 1.5$ (8 to 15$)$ \\
\hline No. of pedicle screws & $16.6 \pm 2.46$ (12 to 23$)$ \\
\hline $\begin{array}{l}\text { No. of pedicle screws per level fused (pedicle } \\
\text { screw density) }\end{array}$ & $1.50 \pm 0.20$ (1.14 to 2.0$)$ \\
\hline No. of other anchors (hooks/wires/clamps) & $1.15 \pm 0.90$ (0 to 5) \\
\hline Preoperative major Cobb angle $\left({ }^{\circ}\right)$ & $55.3 \pm 7.2$ (45 to 80) \\
\hline Supine side bending major Cobb angle $\left({ }^{\circ}\right)$ & $28.0 \pm 10.0$ (1 to 55) \\
\hline Preoperative curve flexibility (\%) & $49.9 \pm 15.2$ (24 to 98$)$ \\
\hline Final postoperative major Cobb angle $\left({ }^{\circ}\right)$ & $18.3 \pm 7.2$ (3 to 36$)$ \\
\hline Absolute degree of Cobb angle correction & $37 \pm 7.1$ (14 to 56$)$ \\
\hline Percent major curve correction & $67.2 \pm 11.7$ (28 to 94) \\
\hline Correction index & $1.45 \pm 0.44$ (0.69 to 2.28$)$ \\
\hline Preoperative T2-T12 kyphosis angle $\left({ }^{\circ}\right)$ & $34.1 \pm 8.8$ (15 to 53$)$ \\
\hline Final postoperative T2-T12 kyphosis angle $\left(^{\circ}\right)$ & $31.6 \pm 8.7(10$ to 50$)$ \\
\hline Change in T2-T12 kyphosis angle $\left({ }^{\circ}\right)$ & $-2.4 \pm 6.9(-19$ to 14$)$ \\
\hline Preoperative rib index & $1.89 \pm 0.29$ (1.25 to 2.58$)$ \\
\hline Final postoperative rib index & $1.6 \pm 0.23$ (1.1 to 2.1$)$ \\
\hline Percent rib index correction & $17.4 \pm 10.1$ (0.1 to 44.5$)$ \\
\hline Loss-correction rate (\%) & $5.08 \pm 6.3$ (0 to 32$)$ \\
\hline Follow-up time (mo) & $40.1 \pm 14.4$ (24 to 92) \\
\hline
\end{tabular}

Values are presented as mean \pm standard deviation (range) or frequency of the patients $(\%)$.

$(p<0.01)$ for an average correction of $67.2 \% \pm 11.7 \%$. The mean preoperative $\mathrm{T} 2-\mathrm{T} 12$ kyphosis angle was $34.1^{\circ} \pm 8.8^{\circ}$ and decreased to $31.6^{\circ} \pm 8.7^{\circ}$ at follow-up $(p<0.01)$. The mean preoperative RI, assessed by thoracic torsion and vertebral rotation, was $1.89 \pm 0.29$ and corrected to $1.6 \pm 0.23$ at follow-up $(p<0.01)$ for an average correction of $17.4 \% \pm 10.1 \%$. There were no significant correlations
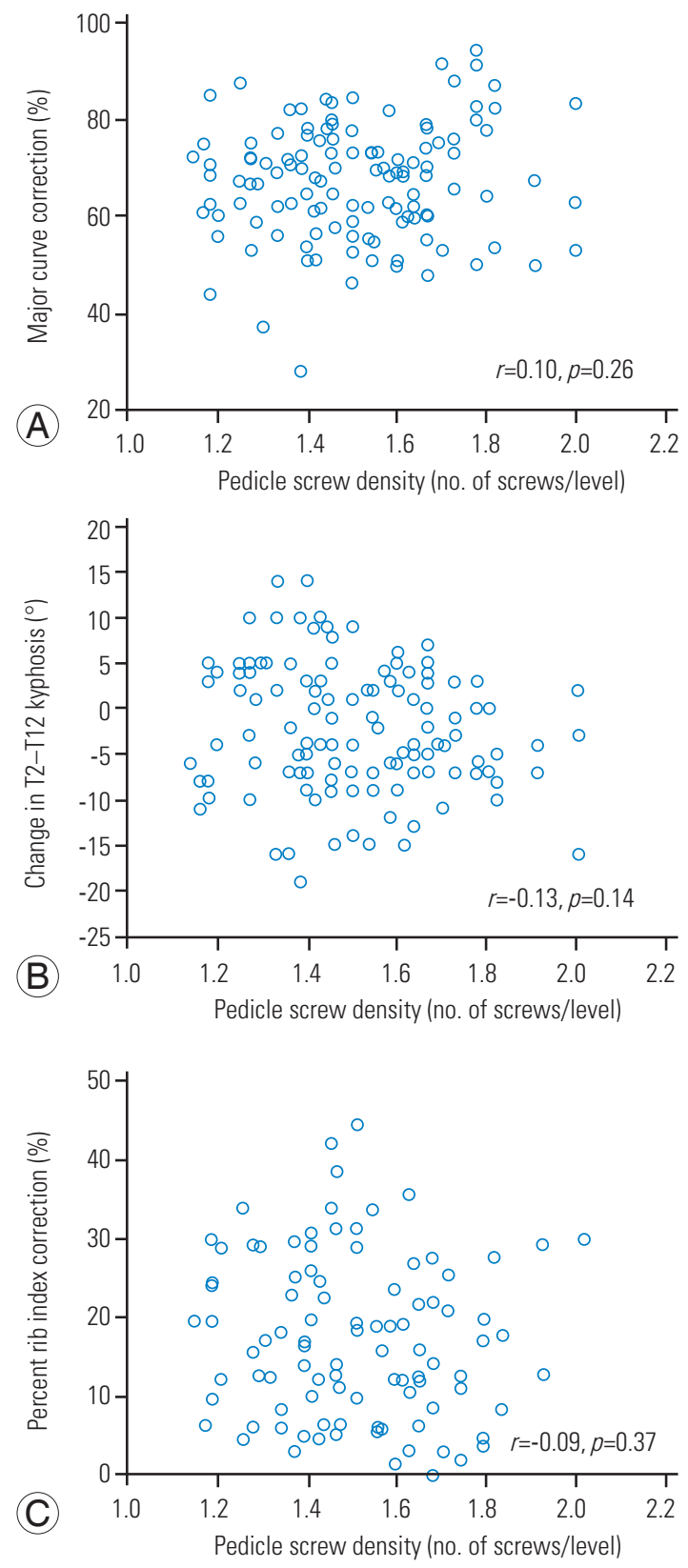

Fig. 4. (A-C) Correlation analysis between pedicle screw density and radiographic outcomes. No significant correlation was found between pedicle screw density and percent major curve correction, change in T2-T12 kyphosis, or percent rib index correction.

between PS density and the following parameters: percent MCC $(r=0.10, p=0.26)$ and CI $(r=0.16, p=0.07)$, change in T2-T12 kyphosis angle $(r=-0.13, p=0.14)$, and percent RI correction $(r=-0.09, p=0.37)$ (Fig. 4$)$. There was no correlation between PS density and absolute degree of Cobb angle correction $(r=0.06, p=0.51)$. Moreover, there was a significant correlation between curve flexibility and percent MCC $(r=0.24, p=0.0086)$. For subgroup correlation 
Table 2. Correlation analyses between pedicle screw density and postoperative radiographic assessment in regards to Lenke curve type

\begin{tabular}{|c|c|c|c|c|}
\hline \multirow{2}{*}{ Lenke curve type } & \multicolumn{2}{|c|}{$\begin{array}{c}\text { Lenke-1 AIS } \\
\text { patients ( } n=99 \text { ) }\end{array}$} & \multicolumn{2}{|c|}{$\begin{array}{c}\text { Lenke- } 2 \text { AIS } \\
\text { patients ( } n=23 \text { ) }\end{array}$} \\
\hline & $r$ & $p$-value & $r$ & $p$-value \\
\hline Percent major curve correction & 0.07 & 0.48 & 0.11 & 0.62 \\
\hline Correction index & 0.15 & 0.14 & 0.27 & 0.18 \\
\hline Change in T2-T12 kyphosis angle $\left({ }^{\circ}\right)$ & -0.09 & 0.37 & -0.28 & 0.20 \\
\hline Percent rib index correction & -0.16 & 0.16 & -0.22 & 0.34 \\
\hline
\end{tabular}

AIS, adolescent idiopathic scoliosis.

Table 3. Correlation analyses between pedicle screw density and postoperative radiographic assessment in regards to patient's age

\begin{tabular}{|c|c|c|c|c|}
\hline \multirow{2}{*}{ Patient's age } & \multicolumn{2}{|c|}{$\begin{array}{l}\text { Patients with age } \\
\quad<15 \text { yr }(n=62)\end{array}$} & \multicolumn{2}{|c|}{$\begin{array}{c}\text { Patients with age } \\
>15 \text { yr }(n=60)\end{array}$} \\
\hline & $r$ & $p$-value & $r$ & $p$-value \\
\hline Percent major curve correction & 0.15 & 0.24 & 0.08 & 0.55 \\
\hline Correction index & 0.19 & 0.12 & 0.03 & 0.80 \\
\hline Change in T2-T12 kyphosis angle $\left(^{\circ}\right)$ & -0.09 & 0.49 & -0.15 & 0.26 \\
\hline Percent rib index correction & -0.06 & 0.68 & -0.15 & 0.29 \\
\hline
\end{tabular}

Table 4. Correlation analyses between pedicle screw density and postoperative radiographic assessment in regards to Risser sign

\begin{tabular}{|c|c|c|c|c|}
\hline \multirow{2}{*}{ Risser sign } & \multicolumn{2}{|c|}{$\begin{array}{l}\text { Risser grade }<3 \\
\qquad(n=55)\end{array}$} & \multicolumn{2}{|c|}{$\begin{array}{c}\text { Risser grade } \geq 3 \\
(n=67)\end{array}$} \\
\hline & $r$ & $p$-value & $r$ & $p$-value \\
\hline Percent major curve correction & 0.12 & 0.39 & 0.08 & 0.47 \\
\hline Correction index & 0.20 & 0.14 & 0.13 & 0.28 \\
\hline Change in T2-T12 kyphosis angle $\left(^{\circ}\right)$ & -0.18 & 0.19 & -0.10 & 0.42 \\
\hline Percent rib index correction & -0.23 & 0.13 & -0.02 & 0.88 \\
\hline
\end{tabular}

Table 5. Correlation analyses between pedicle screw density and postoperative radiographic assessment in regards to body mass index

\begin{tabular}{|c|c|c|c|c|}
\hline \multirow{2}{*}{$\mathrm{BMI}\left(\mathrm{kg} / \mathrm{m}^{2}\right)$} & \multicolumn{2}{|c|}{$\mathrm{BMI}<25(\mathrm{n}=84)$} & \multicolumn{2}{|c|}{$\mathrm{BMI} \geq 25(\mathrm{n}=38)$} \\
\hline & $r$ & $p$-value & $r$ & $p$-value \\
\hline Percent major curve correction & 0.04 & 0.71 & 0.19 & 0.25 \\
\hline Correction index & 0.12 & 0.28 & 0.22 & 0.18 \\
\hline Change in T2-T12 kyphosis angle $\left(^{\circ}\right)$ & -0.16 & 0.15 & -0.04 & 0.79 \\
\hline Percent rib index correction & -0.14 & 0.27 & -0.002 & 0.99 \\
\hline
\end{tabular}

BMI, body mass index.
Table 6. Demographic and preoperative radiographic data for LD and HD groups

\begin{tabular}{|c|c|c|c|}
\hline Variable & $\begin{array}{l}\text { LD group } \\
\qquad(n=57)\end{array}$ & $\begin{array}{l}\text { HD group } \\
(n=65)\end{array}$ & $p$-value \\
\hline Age (yr) & $15.0 \pm 1.95$ & $15.0 \pm 1.82$ & 0.98 \\
\hline Gender (male:female) & $4: 53$ & $12: 53$ & \\
\hline Weight (kg) & $55.8 \pm 13.3$ & $56.4 \pm 13.2$ & 0.79 \\
\hline Height (cm) & $160.3 \pm 8.0$ & $162.2 \pm 10.5$ & 0.31 \\
\hline Body mas index $\left(\mathrm{kg} / \mathrm{m}^{2}\right)$ & $21.1 \pm 3.9$ & $22.5 \pm 5.9$ & 0.18 \\
\hline Risser sign & & & 0.91 \\
\hline Grade 0 & $8(14.0)$ & $6(9.2)$ & \\
\hline Grade 1 & $7(12.3)$ & $12(18.5)$ & \\
\hline Grade 2 & $10(17.5)$ & $12(18.5)$ & \\
\hline Grade 3 & $10(17.5)$ & $12(18.5)$ & \\
\hline Grade 4 & $12(21.1)$ & $12(18.5)$ & \\
\hline Grade 5 & $10(17.5)$ & $11(16.9)$ & \\
\hline Preoperative major Cobb angle $\left({ }^{\circ}\right)$ & $55.5 \pm 7.2$ & $55.1 \pm 7.3$ & 0.77 \\
\hline Preoperative T2-T12 kyphosis angle $\left(^{\circ}\right)$ & $34.7 \pm 9.7$ & $33.5 \pm 7.9$ & 0.49 \\
\hline Preoperative rib index & $1.9 \pm 0.3$ & $1.9 \pm 0.3$ & 0.53 \\
\hline Preoperative curve flexibility (\%) & $50.1 \pm 13.1$ & $49.7 \pm 16.8$ & 0.90 \\
\hline Follow-up time (mo) & $38.0 \pm 14.2$ & $42.0 \pm 14.4$ & 0.13 \\
\hline
\end{tabular}

Values are presented as mean \pm standard deviation or number (\%), unless otherwise stated. No significant between group differences were detected for these variables.

LD, low-screw density; HD, high-screw density.

Table 7. Mean pedicle screws density, number of fused levels, number of pedicle screws and perioperative data for LD and HD groups

\begin{tabular}{lccc} 
Variable & $\begin{array}{c}\text { LD group } \\
\text { (n=57) }\end{array}$ & $\begin{array}{c}\text { HD group } \\
(\mathrm{n}=65)\end{array}$ & $p$-value \\
\hline Mean pedicle screws density & $1.3 \pm 0.1$ & $1.7 \pm 0.1$ & $<0.001^{*}$ \\
\hline No. of fused levels & $11.6 \pm 1.3$ & $10.6 \pm 1.5$ & $<0.001^{*}$ \\
\hline No. of pedicle screws & $15.5 \pm 2.1$ & $17.5 \pm 2.4$ & $<0.001^{*}$ \\
\hline Operative time (min) & $301 \pm 54.0$ & $308 \pm 54.8$ & 0.53 \\
\hline Estimated blood loss (mL) & $586 \pm 354$ & $675 \pm 389$ & 0.21 \\
\hline Rod material & & & 0.78 \\
\hline \multicolumn{1}{c}{ Cobalt chromium } & $16(28.1)$ & $15(23.1)$ & \\
\hline Stainless steel & $26(45.6)$ & $30(46.2)$ & \\
\hline Titanium alloy & $15(26.3)$ & $20(30.8)$ & \\
\hline Rod diameter (mm) & & & 0.53 \\
\hline 6.0 & $16(28.1)$ & $15(23.1)$ & \\
\hline 6.35 & $41(71.9)$ & $50(76.9)$ & \\
\hline
\end{tabular}

Values are presented as mean \pm standard deviation or number $(\%)$, unless otherwise stated.

LD, low-screw density; HD, high-screw density.

${ }^{*} p<0.05$ was considered to be statistical significance. 
Table 8. Comparison of postoperative radiographic data between low-screw density and high-screw density groups

\begin{tabular}{lccc} 
Variable & LD group $(\mathrm{n}=57)$ & HD group (n=65) & $p$-value \\
\hline Postoperative major Cobb angle $\left({ }^{\circ}\right)$ & $18.2 \pm 6.4$ & $18.3 \pm 7.8$ & 0.93 \\
\hline Postoperative T2-T12 kyphosis angle $\left(^{\circ}\right)$ & $33.4 \pm 8.6$ & $30.1 \pm 8.5$ & $0.03^{*}$ \\
\hline Postoperative rib index & $1.5 \pm 0.2$ & $1.6 \pm 0.2$ & 0.15 \\
\hline Percent major curve correction & $67.07 \pm 11.5$ & $67.47 \pm 11.8$ & 0.86 \\
\hline Correction index & $1.4 \pm 0.4$ & $-4(-16$ to 9$)$ & 0.31 \\
\hline Change in T2-T12 kyphosis angle $\left(^{\circ}\right)$ & $-1(-19$ to 14) & $16.5 \pm 8.1$ & 0.13 \\
\hline Percent rib index correction & $18.4 \pm 9.1$ & $5.17(0$ to 32) & 0.36 \\
\hline Loss-correction rate $(\%)$ & $3.33(0$ to 22) & 0.24 \\
\hline
\end{tabular}

Values are presented as mean \pm standard deviation or median (range), unless otherwise stated.

LD, low-screw density; HD, high-screw density.

${ }^{*} p<0.05$ was considered to be statistical significance.

analyses, there were no significant correlations between PS density and the following parameters: percent MCC and CI, change in T2-T12 kyphosis angle, and percent RI correction in regard to Lenke curve type (Lenke curve type 1 and Lenke curve type 2$)$, patient age $(<15$ years and $>15$ years), Risser grade (Risser grade $<3$ and Risser grade $\geq 3$ ), and BMI (BMI $<25 \mathrm{~kg} / \mathrm{m}^{2}$ and $\left.\mathrm{BMI} \geq 25 \mathrm{~kg} / \mathrm{m}^{2}\right)$ (Tables 2-5).

For subgroup analysis, the patients were divided into two groups according to the mean PS density for the entire study. The LD group, consisted of 57 patients, was defined as the use of $<1.50$ screws per fused level, and the HD group, consisted of 65 patients, was defined as the use of 1.50 or more screws per fused level. There were no differences in demographic and preoperative parameters between the two groups (Table 6). The mean PS density was significantly lower in the LD group (1.3 versus 1.7 , $p<0.01)$. The number of fused levels was significantly lower in the HD group (10.6 versus 11.6, $p<0.01$ ). The number of PSs per construct was lower in the LD group (15.5 versus $17.5, p<0.01)$. There were no significant differences in the percentage of rod diameter and rod material use, operative time, and estimated blood loss (Table 7). Statistical subanalysis revealed that there were no significant differences between the HD and LD groups with respect to MCC and CI, change in T2-T12 kyphosis angle, and RI correction. The HD group had less magnitude of postoperative thoracic kyphosis $\left(30^{\circ}\right.$ versus $33^{\circ}, p=0.03$ ) (Table 8). Furthermore, the comparison between patients with PS density $\leq 1.3$ (mean PS density of the entire study-1SD; $\mathrm{n}=21$ ) and patients with PS density $\geq 1.7$ (mean PS density of the entire study+1SD; $n=21$ ) confirmed that there were
Table 9. Postoperative SRS-30 scores

\begin{tabular}{lccc} 
Domain & LD group $^{\mathrm{a}}$ & HD group $^{\mathrm{a})}$ & $p$-value \\
\hline Pain & $3.8 \pm 0.8$ & $4.0 \pm 0.6$ & 0.25 \\
Appearance & $4.0 \pm 0.6$ & $4.0 \pm 0.6$ & 0.98 \\
\hline Function & $3.8 \pm 0.6$ & $3.8 \pm 0.6$ & 0.72 \\
\hline Mental & $4.1 \pm 0.7$ & $4.2 \pm 0.7$ & 0.80 \\
\hline Satisfaction & $4.3 \pm 0.7$ & $4.3 \pm 0.6$ & 0.68 \\
\hline Total score & $3.9 \pm 0.5$ & $4.0 \pm 0.5$ & 0.64 \\
\hline
\end{tabular}

Values are presented as mean \pm standard deviation, unless otherwise stated. No significant between group differences were detected for these variables.

SRS, Scoliosis Research Society; LD, low-screw density; HD, high-screw density.

${ }^{\text {a) }}$ SRS-30 scores were available for 40 patients (70.2\%) out of 57 patients in the LD group and 42 patients (65\%) out of 65 patients in the $\mathrm{HD}$ group.

no significant differences between the two groups in terms of MCC and CI, change in T2-T12 kyphosis angle, and RI correction (all $p>0.05$ ). At the latest follow-up, the SRS-30 scores were available in $82(67.2 \%)$ of 122 patients. SRS30 scores were available in $40(70.2 \%)$ of 57 patients in the low-density group and 42 (65\%) of 65 patients in the high-density group. There were no significant differences in all five domains studied and the overall score between those of the LD and HD groups (Table 9).

No intra- or postoperative instrumentation-related complications were noted. There were three addingon phenomena with required revision surgery in three patients, one patient $(1.8 \%)$ in the LD group and two patients (3.1\%) in the HD group ( $p=1.0)$. In the LD group, an adding-on phenomenon was observed in a 16-yearold girl with an increased fractional curve below the arthrodesis, performed down to T12. A revision procedure 
extending the arthrodesis down to L3 was performed at 2 years after the index procedure. In the HD group, the first adding-on phenomenon was observed in a 14-year-old boy after T3-L1 arthrodesis. The patient underwent extending arthrodesis down to L4 at 1 year after index procedure. In another patient, an 11-year-old girl, an addingon phenomenon was observed after T4-L1 arthrodesis. There was progression of curve at both upper and lower arthrodesis segments. The patient underwent revision extending arthrodesis from T2 to L4 at 3 years after the index procedure. No subsequent complications were noted in all three patients undergoing revision surgery. They were doing well at the latest follow-up. Three patients in the LD group and three patients in the HD group (5.3\% versus $4.6 \%, p=0.72$ ) had curve progression $>5^{\circ}$ at the time of latest follow-up. However, they had no substantial back pain or decline in their function at that time. Their SRS-30 scores, the overall score and all five domains, were $>3$ at the final follow-up. No patients needed additional operation. At the latest follow-up, there were no addingon phenomenon in this cohort. The rates of proximal junctional kyphosis (PJK) were similar between the LD and HD groups (LD group, $8.8 \%$; HD group, $10.8 \%$; $p=0.48$ ) [20]. No patients required revision surgery due to $\mathrm{PJK}$. There was no screw loosening or instrument failure in this study. No patient had secondary operation at the final follow-up.

\section{Discussion}

The present study investigated whether higher PS density improved three-dimensional deformity correction in PSF without POs of flexible, moderate-sized Lenke 1 and 2 AIS. There were no significant correlations between PS density and percent MCC and coronal CI, change in T2-T12 kyphosis angle, and percent RI correction. Furthermore, subgroup analysis confirmed that LD groups had equivalent radiographic and clinical outcomes as HD groups.

We obtained mean percentage MCC of $67.2 \%$ in 122 patients in our cohort, which is comparable to other studies $[8,9,12,13]$. We have considered preoperative curve flexibility, which has been shown to predict curve correction [21]. We have found no correlation between PS density and MCC with or without curve flexibility. Our results were in concordance with the findings of Bharucha et al. [12] and Quan and Gibson [13]. On the contrary,
Clements et al. [8] found a significant correlation between MCC and implant density, but the authors included the patients from all six Lenke curve types and also included variable types of the anchors (wires, hooks, and screws) in their study. In a large multicenter database study, Larson et al. [9] found that increased anchor density was associated with increases in coronal curve correction for Lenke 1 and 2 curves. Unfortunately, this study did not account for preoperative curve flexibility and the use of POs. In recent literature, the use of POs performed in thoracic AIS curves has been investigated and found to significantly improve coronal curve correction $[15,16]$. In the sagittal plane, PS constructs have been shown to provide better thoracic hypokyphotic correction in the previous study [22]. On the contrary, several recent studies have reported that PS constructs decreased magnitude of thoracic kyphosis $[9,10,12,13]$. Additionally, some authors suggested that higher implant density was associated with decreased thoracic kyphosis $[8,9]$. We found loss of thoracic kyphosis in the entire cohort postoperatively. However, the magnitude of postoperative kyphosis was in physiologic range. Furthermore, the loss of preoperative to postoperative kyphosis was equivalent between HD and LD groups. We believe that this may be due to the hypokyphotic nature of the deformity in adolescent idiopathic thoracic scoliosis. Thoracic kyphosis alignment is sacrificed to achieve more coronal plane correction [13].

There are very few evidences on vertebral rotation and rib hump deformity correction regarding PS density in scoliotic treatment. PS constructs have been shown to improve vertebral rotation correction and lessen rib hump deformity compared with hook-rod instrumentation [23]. Kuklo et al. [24] assessed the correlation between rib hump deformity and apical vertebral rotation and found the good and high significant correlations between these two parameters. We used rib hump, in terms of RI method, to measure vertebral rotation for patients who did not undergo costoplasty. We found no significant correlation between vertebral rotation, in terms of RI correction, and PS density. Our results are supported by previous studies. Yang et al. [25] evaluated vertebral rotation correction indirectly from photographs of the rib hump. The authors found no significant correlation between implant density and vertebral rotation assessed by photographic parameters. Bharucha et al. [12] evaluated thoracic angle of trunk rotation using a scoliometer. They also found no correlation between implant density and thoracic angle of trunk 
rotation at 2 years postoperatively.

The present study also reported the change in percent MCC from immediate postoperative radiographs to final follow-up with an average loss of correction of $5 \%$ for the entire cohort. Our results are similar to the previous studies, which reported loss of correction rate ranging from $2 \%$ to $5 \%$ in patients with AIS who underwent PSF with PS constructs [26-28].

In this study, we focused on three-dimensional deformity correction in PSF without the use of POs in thoracic idiopathic scoliosis. All patients underwent a similar posterior surgical release procedure. The exposure was performed with a midline incision and extending to the tips of transverse process. No additional posterior surgical release was employed. We confined the anchor type of the study groups that used only predominantly PS constructs, and all patients only had thoracic scoliotic curve (Lenke 1 and 2 curve types) to render homogeneity of the study. We also accounted for the influence of flexibility on the coronal correction. Our results are supported by previous studies by Cheung et al. [21] and Quan et al. [13]. As regard the biomechanical perspective, Wang et al. [29] and Le Naveaux et al. [30] revealed that low-screw density construct provided similar three-dimensional correction as the higher-screw density construct among patients who underwent PSF for thoracic AIS. Finally, we believe that implant density at some specific regions of the construct, i.e., at the apex and both ends, may be more crucial than overall implant density for three-dimensional deformity correction in thoracic AIS [31,32].

This study has some limitations. First, its retrospective nature makes it vulnerable to various biases. A randomization of anchor density would more adequately address the confounding factors especially for surgeon bias. Second, four surgeons were involved, and the correction techniques were not standardized. Further studies should take into account for identical correction technique so as to remove this confounding factor. Third, our sample size was small. This decreased the power for statistical analysis and undermines the conclusion that there was no correlation between PS density and amount of correction. Fourth, the problem within the fusion area (such as failure of fixation or pseudarthrosis) is one of the major concerns following AIS surgery and may affect surgical outcomes. However, we did not implement the methods and results for evaluating the fusion status in this study. Further study should evaluate the fusion rate or pseudarthrosis after AIS surgery. Although not the primary objective of this study, one of the important issues for thoracic AIS correction is shoulder imbalance correction. However, a previous study found no correlation between increased implant density and shoulder balance correction [25]. The correlation between implant density and shoulder imbalance needs to be further assessed in the future. Finally, the majority (54.9\%) of patients in our cohort has Risser grade $\geq 3$ so this may limit the generalizability of our results for patients with less skeletal maturity.

\section{Conclusions}

Our study showed that there was no significant correlation between PS density and amount of coronal, sagittal, or axial plane correction. LD constructs may be used as effectively as HD constructs for patients with flexible, moderate-sized thoracic AIS undergoing PSF without the use of POs.

\section{Conflict of Interest}

No potential conflict of interest relevant to this article was reported.

\section{Acknowledgments}

The authors would like to thank Donita Bylski-Austrow, PhD (Division of Orthopaedic Surgery, Cincinnati Children's Hospital Medical Center, Cincinnati, $\mathrm{OH}$ ) and Sasivimol Rattanasiri, PhD (Section for Clinical Epidemiology and Biostatistics, Mahidol University, Bangkok, Thailand) for statistical analysis assistance.

\section{Author Contributions}

All authors conceptualized and designed the study. Thamrong Lertudomphonwanit collected and analyzed the data, drafted and revised the manuscript. Chirag A. Berry was responsible for collection and analysis data. Peter F. Sturm helped to draft the manuscript. Viral V. Jain helped to draft and revise the manuscript. All authors read and approved the final manuscript.

\section{References}

1. Lenke LG, Kuklo TR, Ondra S, Polly DW Jr. Ratio- 
nale behind the current state-of-the-art treatment of scoliosis (in the pedicle screw era). Spine (Phila Pa 1976) 2008;33:1051-4.

2. Kadoury S, Cheriet F, Beausejour M, Stokes IA, Parent $\mathrm{S}$, Labelle $\mathrm{H}$. A three-dimensional retrospective analysis of the evolution of spinal instrumentation for the correction of adolescent idiopathic scoliosis. Eur Spine J 2009;18:23-37.

3. Kim YJ, Lenke LG, Cho SK, Bridwell KH, Sides B, Blanke K. Comparative analysis of pedicle screw versus hook instrumentation in posterior spinal fusion of adolescent idiopathic scoliosis. Spine (Phila Pa 1976) 2004;29:2040-8.

4. Di Silvestre M, Bakaloudis G, Lolli F, Vommaro F, Martikos K, Parisini P. Posterior fusion only for thoracic adolescent idiopathic scoliosis of more than 80 degrees: pedicle screws versus hybrid instrumentation. Eur Spine J 2008;17:1336-49.

5. Yilmaz G, Borkhuu B, Dhawale AA, et al. Comparative analysis of hook, hybrid, and pedicle screw instrumentation in the posterior treatment of adolescent idiopathic scoliosis. J Pediatr Orthop 2012;32:490-9.

6. Dobbs MB, Lenke LG, Kim YJ, Luhmann SJ, Bridwell $\mathrm{KH}$. Anterior/posterior spinal instrumentation versus posterior instrumentation alone for the treatment of adolescent idiopathic scoliotic curves more than 90 degrees. Spine (Phila Pa 1976) 2006;31:2386-91.

7. Kuklo TR, Potter BK, Lenke LG, Polly DW Jr, Sides B, Bridwell KH. Surgical revision rates of hooks versus hybrid versus screws versus combined anteroposterior spinal fusion for adolescent idiopathic scoliosis. Spine (Phila Pa 1976) 2007;32:2258-64.

8. Clements DH, Betz RR, Newton PO, Rohmiller M, Marks MC, Bastrom T. Correlation of scoliosis curve correction with the number and type of fixation anchors. Spine (Phila Pa 1976) 2009;34:2147-50.

9. Larson AN, Polly DW Jr, Diamond B, et al. Does higher anchor density result in increased curve correction and improved clinical outcomes in adolescent idiopathic scoliosis? Spine (Phila Pa 1976) 2014;39:571-8.

10. Ketenci IE, Yanik HS, Demiroz S, Ulusoy A, Erdem S. Three-dimensional correction in patients with Lenke 1 adolescent idiopathic scoliosis: comparison of consecutive versus interval pedicle screw instrumentation. Spine (Phila Pa 1976) 2016;41:134-8.
11. Suk SI, Kim WJ, Lee SM, Kim JH, Chung ER. Thoracic pedicle screw fixation in spinal deformities: are they really safe? Spine (Phila Pa 1976) 2001;26:204957.

12. Bharucha NJ, Lonner BS, Auerbach JD, Kean KE, Trobisch PD. Low-density versus high-density thoracic pedicle screw constructs in adolescent idiopathic scoliosis: do more screws lead to a better outcome? Spine J 2013;13:375-81.

13. Quan GM, Gibson MJ. Correction of main thoracic adolescent idiopathic scoliosis using pedicle screw instrumentation: does higher implant density improve correction? Spine (Phila Pa 1976) 2010;35:5627.

14. Chen J, Yang C, Ran B, et al. Correction of Lenke 5 adolescent idiopathic scoliosis using pedicle screw instrumentation: does implant density influence the correction? Spine (Phila Pa 1976) 2013;38:E946-51.

15. Takahashi J, Ikegami S, Kuraishi S, Shimizu M, Futatsugi T, Kato H. Skip pedicle screw fixation combined with Ponte osteotomy for adolescent idiopathic scoliosis. Eur Spine J 2014;23:2689-95.

16. Pizones J, Sanchez-Mariscal F, Zuniga L, Izquierdo E. Ponte osteotomies to treat major thoracic adolescent idiopathic scoliosis curves allow more effective corrective maneuvers. Eur Spine J 2015;24:1540-6.

17. Lenke LG, Edwards CC 2nd, Bridwell KH. The Lenke classification of adolescent idiopathic scoliosis: how it organizes curve patterns as a template to perform selective fusions of the spine. Spine (Phila Pa 1976) 2003;28:S199-207.

18. Grivas TB, Dangas S, Polyzois BD, Samelis P. The Double Rib Contour Sign (DRCS) in lateral spinal radiographs: aetiologic implications for scoliosis. Stud Health Technol Inform 2002;88:38-43.

19. Vora V, Crawford A, Babekhir N, et al. A pedicle screw construct gives an enhanced posterior correction of adolescent idiopathic scoliosis when compared with other constructs: myth or reality. Spine (Phila Pa 1976) 2007;32:1869-74.

20. Kim YJ, Lenke LG, Bridwell KH, et al. Proximal junctional kyphosis in adolescent idiopathic scoliosis after 3 different types of posterior segmental spinal instrumentation and fusions: incidence and risk factor analysis of 410 cases. Spine (Phila Pa 1976) 2007;32:2731-8.

21. Cheung KM, Natarajan D, Samartzis D, Wong YW, 
Cheung WY, Luk KD. Predictability of the fulcrum bending radiograph in scoliosis correction with alternate-level pedicle screw fixation. J Bone Joint Surg Am 2010;92:169-76.

22. Suk SI, Kim WJ, Kim JH, Lee SM. Restoration of thoracic kyphosis in the hypokyphotic spine: a comparison between multiple-hook and segmental pedicle screw fixation in adolescent idiopathic scoliosis. J Spinal Disord 1999;12:489-95.

23. Asghar J, Samdani AF, Pahys JM, et al. Computed tomography evaluation of rotation correction in adolescent idiopathic scoliosis: a comparison of an all pedicle screw construct versus a hook-rod system. Spine (Phila Pa 1976) 2009;34:804-7.

24. Kuklo TR, Potter BK, Lenke LG. Vertebral rotation and thoracic torsion in adolescent idiopathic scoliosis: what is the best radiographic correlate? J Spinal Disord Tech 2005;18:139-47.

25. Yang S, Jones-Quaidoo SM, Eager M, et al. Right adolescent idiopathic thoracic curve (Lenke $1 \mathrm{~A}$ and B): does cost of instrumentation and implant density improve radiographic and cosmetic parameters? Eur Spine J 2011;20:1039-47.

26. Cheng I, Kim Y, Gupta MC, et al. Apical sublaminar wires versus pedicle screws: which provides better results for surgical correction of adolescent idiopathic scoliosis? Spine (Phila Pa 1976) 2005;30:2104-12.
27. Kim YJ, Lenke LG, Kim J, et al. Comparative analysis of pedicle screw versus hybrid instrumentation in posterior spinal fusion of adolescent idiopathic scoliosis. Spine (Phila Pa 1976) 2006;31:291-8.

28. Hwang CJ, Lee CK, Chang BS, Kim MS, Yeom JS, Choi JM. Minimum 5-year follow-up results of skipped pedicle screw fixation for flexible idiopathic scoliosis. J Neurosurg Spine 2011;15:146-50.

29. Wang X, Aubin CE, Robitaille I, Labelle H. Biomechanical comparison of alternative densities of pedicle screws for the treatment of adolescent idiopathic scoliosis. Eur Spine J 2012;21:1082-90.

30. Le Naveaux F, Larson AN, Labelle H, Wang X, Aubin CE. How does implant distribution affect 3D correction and bone-screw forces in thoracic adolescent idiopathic scoliosis spinal instrumentation? Clin Biomech (Bristol, Avon) 2016;39:25-31.

31. Lertudomphonwanit T, Jain VV, Sturm PF, Patel S. Periapical-dropout screws strategy for 3-dimensional correction of Lenke 1 adolescent idiopathic scoliosis in patients treated by posterior spinal fusion. Clin Spine Surg 2019;32:E359-65.

32. Delikaris A, Wang X, Boyer L, Larson AN, Ledonio CG, Aubin CE. Implant density at the apex is more important than overall implant density for 3D correction in thoracic adolescent idiopathic scoliosis using rod derotation and en bloc vertebral derotation technique. Spine (Phila Pa 1976) 2018;43:E639-47. 